\title{
RELATION BETWEEN NEONATAL ENDOTRACHEAL (ET) TUBE SIZE AND AEROSOL PENETRATION- COMPUTATIONAL FLUID DYNAMIC STUDY (CFD)
}

\author{
J. Mazela ${ }^{1}$, A. Moskal $^{2}$, T. Sosonowski ${ }^{2}$, J. Gadzinowski $^{1}$ \\ ${ }^{l}$ Neonatology, Poznan University of Medical Sciences, Poznan, ${ }^{2}$ Chemical and Process Engineering, \\ Warsaw University of Technology, Warsaw, Poland
}

Introduction: There are several aerosolized drugs used in the treatment of neonatal respiratory illnesses, including bronchodilators, diuretics and steroids. In vitro and in vivo studies identified variables that affect aerosol efficiency, including particle size, aerosol flows, and nebulizer choice. Type of patient interface can also be a critical variable in determining particle size of therapeutic aerosols delivered to mechanically ventilated patients. Unfortunately there are limited data on the influence of neonatal ET tube size on aerosol characteristics and lung inhaled and deposited dose in in vivo studies.

Aim: To establish a relationship between neonatal ET tube size and penetration of aerosols with different particle sizes and flow characteristics.

Methods: Three different ET tube sizes were tested: 2.5; 3.0 and $3.5 \mathrm{~mm}$. Computations of the airflow field and aerosol particle dynamics during realistic inhalations have been performed with the commercial CFD package Fluent (Fluent, Inc.) as described earlier. The simulated breathing curves were based on two different inspiratory flows: 3 and $5 \mathrm{~L} / \mathrm{min}$. Penetration \% was calculated based on spherical monodisperse aerosol particles with MMAD ranging from 1 to $10 \mu \mathrm{m}$.

Results: 50\% penetration was achieved only for 3.5 ET tube for all range of particle size. There was lower penetration profile for higher inspiratory flows.

Conclusions: ET tube size can significantly influence the inhaled therapeutic dose by limiting aerosol ET tube penetration for non optimized particle size of the aerosols given to infants with breathing support. CFD can be very useful tool for designing optimal aerosol delivery system. 